\title{
Plaquetograma em Gestantes Normais e com Pré-eclâmpsia
}

\author{
Measurement of Platelet Parameters in Normal and Preeclamptic Pregnant Women
}

Elvany Verônica dos Santos, José Meirelles Filho

\section{RESUMO}

\begin{abstract}
Objetivo: avaliar parâmetros do plaquetograma comparando os valores em gestantes normais e com pré-eclâmpsia.

Métodos: realizou-se estudo transversal controlado. Foram revisados os prontuários das mulheres internadas em hospital universitário, no periodo de 1 de janeiro de 2001 a 31 de julho de 2002. Foram pré-selecionadas aquelas que tinham plaquetograma realizado a partir da $28^{a}$ semana de gravidez. Foram analisados dois grupos de estudo: grupo PE (36 portadoras de pré-eclâmpsia) e grupo GN (58 gestantes normais). Os parâmetros plaquetários analisados pelo método automatizado foram: contagem de plaquetas, volume médio de plaquetas (MPV), largura de distribuição de plaquetas (PDW) e razão de células grandes de plaquetas (P-LCR). A análise estatística usou o teste t de Student e o teste do $\chi^{2}$ para comparar os grupos, e para avaliar o grau de dependência entre as variáveis utilizou-se o coeficiente de determinação $\left(r^{2}\right)$. Para todos os testes, o nivel de significância considerado foi $p \leq 0,05$.

Resultados: a contagem de plaquetas não foi diferente entre os grupos, porém os demais parâmetros plaquetários estavam significativamente mais elevados no grupo PE. A gravidade da doença foi documentada em 91,7\% das portadoras de pré-eclâmpsia, apesar de nenhuma das pacientes incluidas ter apresentado trombocitopenia como critério de gravidade. Detectaram-se correlações negativas entre a contagem das plaquetas e entre os demais parâmetros plaquetários analisados, e correlações positivas entre MPV e PDW, MPV e P-LCR e entre PDW e P-LCR. Observaram-se ainda correlações positivas entre MPV, PDW e P-LCR e as pressões sistólicas e diastólicas máximas.

Conclusões: a pré-eclâmpsia esteve associada com estes novos parâmetros plaquetários, sugerindo alterações funcionais das plaquetas. A aplicabilidade clínica destes parâmetros, como marcadores mais precoces de gravidade da pré-eclâmpsia, exige mais estudos.
\end{abstract}

PALAVRAS-CHAVE: Hipertensão. Pré-eclâmpsia. Plaquetas. Trombocitopenia.

\section{Introdução}

Especulam-se quais sejam os mecanismos fisiopatológicos que resultam no desenvolvimento de hipertensão em gestantes saudáveis, ou o agravamento desta em mulheres previamente

Departamento de Ginecologia e Obstetrícia

Hospital Universitário Júlio Müller

Faculdade de Ciências Médicas

Universidade Federal de Mato Grosso

Correspondência:

José Meirelles Filho

Avenida Ipiranga, 280 - apto. 1701 - Goiabeiras

78020-030 - Cuiabá - MT

e-mail: jmf@terra.com.br

Tel.: (65) 612-4196; 615-7330 hipertensas. Há considerável interesse no papel que as plaquetas desenvolvem na patogênese da pré-eclâmpsia $(\mathrm{PE})$ e no fato de a ativação e o consumo plaquetários ocorrerem no desenvolvimento desta sindrome. Queda precoce no número de plaquetas foi detectada em mulheres com hipertensão crônica que vieram a desenvolver préeclâmpsia superposta, quando comparadas às que não desenvolveram a doença. Isto sugere que o aumentado consumo de plaquetas é achado precoce, precedendo outros aspectos da doença ${ }^{1}$.

Redman $^{2}$ destacou a importância da ativação plaquetária, levantou a hipótese de que a vasoconstrição que causa a hipertensão pode ter a mesma origem que a ativação das plaquetas e 
concluiu que mais estudos envolvendo as plaquetas poderiam esclarecer a patogênese da doença. Após uma década do destaque dado por este autor às plaquetas, pesquisadores suecos reafirmaram que a causa exata da pré-eclâmpsia persiste sem elucidação. Contudo, evidências demonstram papel relevante das plaquetas na patogênese da doença ${ }^{3}$.

À avaliação sistemática das plaquetas pelos contadores eletrônicos de múltiplos canais, onde são analisados vários parâmetros plaquetários, justifica-se aplicar o termo plaquetograma, em analogia às demais subdivisões do hemograma. Estes instrumentos contam as plaquetas por tecnologia de impedância ou dispersão de $l u z^{4,5}$. A contagem normal de plaquetas por contadores eletrônicos é exata e reprodutível, com erro sistemático menor que $10 \%$ para contagem maior que $70.000 / \mathrm{UI}$ e insatisfatória para contagens inferiores, pois o erro aumenta com a trombocitopenia ${ }^{6}$. Desde o advento da contagem sangüinea automatizada, a trombocitopenia (contagem de plaquetas inferior a $150.000 / \mathrm{mm}^{3}$ ) é detectada, freqüentemente, em mulheres grávidas assintomáticas ${ }^{7}$.

É bem aceito que as plaquetas são heterogêneas com respeito ao volume e à densidade. Entre os novos índices plaquetários, vem merecendo destaque o volume plaquetário médio (MPV), por se tratar de variável biológica que determina a função e atividade plaquetária ${ }^{8}$. A avaliação do volume plaquetário tornou-se mais constante, após o advento de contadores hematológicos modernos, porém as alterações do MPV não estão suficientemente esclarecidas ${ }^{6,9,10}$. A importância clínica de volumes plaquetários maiores tem sido relacionada com a presença de agregação plaquetária aumentada $^{3,8,11}$.

Recentemente, investigadores demonstraram consideráveis alterações plaquetárias na préeclâmpsia. Confirmaram a diminuição significante do número de plaquetas, mas os elevados valores do volume médio de plaquetas e distúrbio na distribuição de densidade das plaquetas só foram observados em subgrupo de PE classificado como grave ${ }^{3}$.

Além do volume médio das plaquetas, os contadores hematológicos mais modernos fornecem parâmetros para o estudo da morfologia das plaquetas. A largura da distribuição das plaquetas (PDW) é o coeficiente de variação do histograma que reflete as amplitudes da variação das populações plaquetárias ${ }^{6}$. O volume médio de plaquetas é o parâmetro mais estudado, ao passo que para o PDW há escassas citações científicas ${ }^{11}$. O MPV e o PDW são expressos em fentolitros $\left(1 \mathrm{fL}=10^{-15} \mathrm{li}\right.$ - tros) enquanto o P-LCR é expresso em porcentagem.

Estudos mais recentes trazem referências destes parâmetros plaquetários, sugerindo alterações que podem justificar sua aplicabilidade clini$\mathrm{ca}^{3,8,11,12}$. A razão das células grandes de plaquetas (P-LCR), é a razão de grandes plaquetas encontradas entre os limites fixo que é de 12 fentolitros (fL), usado no intuito de ser um indicador, e o superior, na faixa de 12 a $30 \mathrm{fL}$. Ela é calculada dividindo-se o número de células que se encontram na faixa entre os limites fixo e o superior pelo numero de células que se encontram na faixa entre os limites inferior e superior. O limite inferior é ajustado automaticamente na faixa de 2 a $6 \mathrm{fL}$.

Diante da relevância da $\mathrm{PE}$, associada à escassez de trabalhos sobre as alterações dos parâmetros plaquetários, achamos adequada a proposição de analisar o possivel papel desses novos parâmetros nas gestações complicadas com PE.

\section{Pacientes e Métodos}

Trata-se de estudo retrospectivo, do tipo transversal controlado, em que se propõe avaliar o plaquetograma em gestantes normais e com préeclâmpsia.

A partir de levantamento dos arquivos do registro de partos do Centro Obstétrico do Hospital Universitário Júlio Müller (HUJM) - Cuiabá/MT, foram revisados 835 prontuários das mulheres que pariram no período de 1 de janeiro de 2001 a 31 de julho de 2002. Foram pré-selecionas aquelas que tinham documentado plaquetograma realizado a partir da $28^{\mathrm{a}}$ semana de gravidez. De acordo com os critérios de elegibilidade pré-estabelecidos para casos e controles, foram elegiveis e incluídas no estudo 94 mulheres. Estas foram separadas em dois grupos: grupo PE (36 portadoras de PE) e grupo GN (58 gestantes normais).

Os critérios de elegibilidade foram: para o grupo PE: pressão sanguínea $\geq 140 / 90 \mathrm{mmHg}$ (pelo menos duas medidas), após a $28^{\mathrm{a}}$ semana de gravidez, excluindo o período de trabalho de parto, associada à proteinúria $\geq 300 \mathrm{mg}$ em urina de 24 horas ou Labstix (+) ou mais em amostra casual; não terem feito uso na gestação de medicamentos (exceto aqueles usados para controle da hipertensão), não apresentarem doenças associadas e gestação única. Para o grupo GN: proteinúria não significativa em amostra casual, ausência de doenças associadas e terem gestação única, além de parto a termo, recém-nascido sem crescimento intra-uterino restrito ou baixo peso ao nascer, 
e sem malformações congênitas. Não terem feito uso durante a gestação de medicamentos que pudessem alterar parâmetros plaquetários complementava os critérios.

A idade gestacional teve como base o método de Capurro, tendo em vista a incerteza da data da última menstruação (DUM), por parte de algumas mulheres, e/ou a não realização de ultrasonografia gestacional precoce. No grupo PE foram considerados os resultados de exames laboratoriais mais próximos ao parto. Os parâmetros plaquetários analisados foram: a contagem das plaquetas, o MPV, a PDW, e a razão de células grandes das plaquetas (P-LCR). Todas as determinações foram realizadas em equipamento analisador hematológico computadorizado completamente automatizado (Sysmex SF-3000, Roche) para uso no diagnóstico in vitro, em laboratórios clínicos. O número de plaquetas foi determinado pelo bloco de detector RBC (célula vermelha do sangue) usando o método de corrente contínua para detecção. As análises dos indices MPV, PDW e P-LCR foram determinadas pelo método automatizado.

A proteinúria foi apurada de duas maneiras: por meio de coleta de 24 horas ou por amostra casual. O equipamento utilizado foi o analisador automatizado (Cobas Mira Plus, Roche) ou fita (Labstix), respectivamente. Como proteinúria significativa, consideraram-se os valores maiores ou iguais a $300 \mathrm{mg}$ em 24 horas ou $30 \mathrm{mg} / \mathrm{dL}$ ou Labstix de $(+)$ ou mais em amostra casual.

A pré-eclâmpsia foi considerada grave quando estivesse presente um ou mais dos seguintes critérios: pressão arterial diastólica igual/maior que $110 \mathrm{mmHg}$; proteinúria igual/maior que 2,0 g/L em 24 horas; oligúria (menor que $500 \mathrm{~mL}$ / dia, ou $15 \mathrm{~mL} /$ hora); niveis séricos de creatinina maiores que $1,2 \mathrm{mg} / \mathrm{dL}$; sinais de encefalopatia hipertensiva; sinais de insuficiência cardiaca; dor epigástrica ou em hipocôndrio direito; plaquetopenia $\left(<100.000 / \mathrm{mm}^{3}\right)$; aumento de enzimas hepáticas (AST ou TGO, ALT ou TGP, DHL) e de bilirrubinas; presença de esquizócitos em esfregaço de sangue periférico; presença de crescimento intra-uterino retardado e/ ou oligoâmnio e evidência clínica e/ou laboratorial de coagulopatia ${ }^{13}$.

A gravidade da pré-eclâmpsia foi determinada segundo os critérios de gravidade estabelecidos pelo Colégio Americano de Obstetras e Ginecologistas (ACOG), referidos no Manual de Gestação de Alto Risco do Ministério da Saúde ${ }^{13}$.

Os dados foram coletados utilizando formulários padronizados e armazenados em banco de dados do programa Epi-Info, versão 6.04, calculan- do-se as médias com os respectivos desvios-padrão das variáveis continuas e distribuição de freqüência de variáveis categóricas. O teste $t$ de Student foi utilizado para testar diferenças entre médias quando as variáveis tinham distribuição normal. Para avaliar o grau de dependência entre as variáveis continuas utilizou-se o coeficiente de determinação $\left(\mathrm{r}^{2}=\right.$ quadrado do coeficiente de correlação de Pearson). $\mathrm{O} \mathrm{r}^{2}$ é interpretado como a porcentagem de variação explicada por uma das variáveis em relação à outra e expresso em porcentagem. Para todos os testes, o nivel de significância considerado foi $\mathrm{p} \leq 0,05$.

O projeto de pesquisa deste estudo foi analisado e aprovado pelo Comitê de Ética em Pesquisa do HUJM, da Universidade Federal de Mato Grosso. Não há conflitos de interesses. Os dados foram coletados dos prontuários das mulheres incluídas no estudo e as análises realizadas, à época, faziam parte da rotina do serviço, no atendimento às grávidas normais e às complicadas com pré-eclâmpsia.

\section{Resultados}

A média de idade das participantes foi de $24,6 \pm 6,2$ anos e 23,8 $\pm 5,0$ anos para os grupos PE e GN, respectivamente, sem diferença significativa entre os grupos $(p=0,457)$. Houve maior proporção de primíparas no grupo PE (50 vs $22,4 \%$ ), sendo a diferença significativa $(p=0,018)$.

Observou-se que $77,8 \%$ das portadoras de $\mathrm{PE}$ apresentaram niveis de gravidade, considerandose a pressão sistólica máxima, e $72,2 \%$ quando se considerou a pressão diastólica máxima, (PAS $\geq 160$ $\mathrm{mmHg}$ e $\mathrm{PAD} \geq 110 \mathrm{mmHg}$ ).

A média da proteinúria foi de $2,3 \pm 1,6 \mathrm{~g} / 24 \mathrm{~h}$ no grupo $\mathrm{PE}$, confirmando a gravidade dos casos. A maioria das portadoras de PE estava classificada como grave, além de um caso de eclâmpsia $(91,7 \%)$. Três dos casos tiveram a síndrome HELLP, só confirmada no pós-parto imediato.

As médias da contagem das plaquetas foram de $211,1 \pm 70,1 \mathrm{~mL} / \mathrm{mm}^{3}$ e de $229,3 \pm 61,6 \mathrm{~mL} / \mathrm{mm}^{3}$ nos grupos PE e GN, respectivamente, porém esta diferença não foi significativa (Tabela 1). Observou-se trombocitopenia em $19,4 \%$ no grupo $\mathrm{PE}$ e em $6,9 \%$ no grupo GN, sem diferença significativa $(p=0,1391)$. Das 36 portadoras de pré-eclâmpsia nenhuma apresentou contagem de plaquetas inferior a $100.000 / \mathrm{mm}^{3}$, embora três casos com sindrome HELLP tenham sido registrados. O fato se deve à queda no número de plaquetas só ter ocorrido no pós-parto imediato. 
Tabela 1 - Médias, desvios padrão e faixas de variação dos parâmetros plaquetários nas portadoras de pré-eclâmpsia (PE) e nas gestantes normais (GN).

\begin{tabular}{lccc}
\hline Parâmetros plaquetários & PE & GN & p \\
\hline PLT $\left(10^{3} / \mathrm{mm}^{3}\right)$ & $211,1 \pm 70,1$ & $229,3 \pm 61,6$ & 0,191 \\
Variação & $(102-363)$ & $(124-362)$ & \\
PDW (fL) & $13,6 \pm 2,7$ & $12,2 \pm 2,1$ & $0,005^{*}$ \\
Variação & $(9,2-21,5)$ & $(8,5-16)$ & \\
MPV(fL) & $11,0 \pm 1,2$ & $10,3 \pm 1,2$ & $0,008^{*}$ \\
Variação & $(8,4-14,0)$ & $(8-12,7)$ & \\
P-LCR (\%) & $33,4 \pm 9,2$ & $28,4 \pm 8,8$ & $0,011^{*}$ \\
Variação & $(14,1-54,9)$ & $(10,9-46,7)$ & \\
\hline
\end{tabular}

Teste $t$ de Student; * diferença significativa $(\mathrm{p} \leq 0,05)$

$\mathrm{PLT}=$ contagem de plaquetas; $\quad \mathrm{MPV}=$ volume médio das plaquetas

PDW = largura de distribuição das plaquetas; P-LCR = razão de células grandes de plaquetas.

$\mathrm{fL}=$ fentolitro

Não se demonstrou associação entre a contagem de plaquetas e a PE. Por outro lado, associações entre PE e todos os outros parâmetros plaquetários (MPV, PDW e P-LCR) foram demonstradas. Os valores encontrados foram significativamente mais elevados no grupo $\mathrm{PE}$ quando comparados aos do grupo GN (Tabela 1).

Os resultados das correlações entre os parâmetros plaquetários entre si e também com as médias das PAS e PAD máximas estão demonstrados na Tabela 2. Correlações negativas entre a contagem de plaquetas e os demais parâmetros plaquetários foram demonstradas no presente estudo. Observamos correlações positivas fortes entre MPV e PDW entre MPV e P-LCR e entre PDW e P-LCR. A correlação positiva forte entre o MPV e a P-LCR é a mais expressiva $\left(r^{2}=0,96 ; p<0,05\right)$, sendo de $96 \%$ a influência do volume médio sobre a razão de células grandes.

Tabela 2 - Correlações dos índices plaquetários entre si e de cada índice com as médias nos acréscimos dos níveis das pressões sistólicas e diastólicas máximas nas portadoras de PE e nas gestantes normais.

\begin{tabular}{lcccccc}
\hline Variável & PASmax & PADmax & PLT & PDW & MPV & P-LCR \\
\hline PLT & $-0,11$ & $-0,06$ & - & $-0,39^{*}$ & $-0,35^{*}$ & $-0,38^{*}$ \\
PDW & $0,28^{*}$ & $0,25^{*}$ & $-0,39^{*}$ & - & $0,90^{*}$ & $0,93^{*}$ \\
MPV & $0,26^{*}$ & $0,26^{*}$ & $-0,35^{*}$ & $0,90^{*}$ & - & $0,96^{*}$ \\
P-LCR & $0,25^{*}$ & $0,24^{*}$ & $-0,38^{*}$ & $0,93^{*}$ & $0,96^{*}$ & - \\
\hline
\end{tabular}

$\mathrm{r}^{2}=$ coeficiente de determinação; * $\mathrm{p} \leq 0,05$ significativo

$\mathrm{PLT}=$ contagem de plaquetas; $\mathrm{MPV}=$ volume médio das plaquetas; $\mathrm{PASmax}$ = acréscimo no nível de pressão arterial sistólica máxima; PADmax = acréscimo do nível de pressão arterial diastólica máxima; PDW = largura de distribuição das plaquetas; P-LCR = razão de células grandes de plaquetas.

Não houve correlação significativa entre a contagem das plaquetas e o acréscimo dos níveis da PADmax ou PASmax ( $p>0,05)$. As correlações positivas entre os outros parâmetros plaquetários e os niveis pressóricos diastólicos e sistólicos máximos, apesar de fracas, se mostraram significativas. Com relação à correlação entre MPV e PASmax, apesar de fraca, mostrou-se significativa $\left(r^{2}=0,26 ; p<0,05\right)$. O acréscimo dos niveis da PASmax representa $26 \%$ de influência desta variável sobre o aumento do MPV.

\section{Discussão}

A etiologia da pré-eclâmpsia persiste desconhecida, desafiando estudiosos e pesquisadores. Redman $^{2}$ acreditava ser a doença dependente do trofoblasto e prevenida, pelo menos em parte, por drogas antiplaquetárias. No presente trabalho, detectou-se trombocitopenia em 19,9\% do grupo $\mathrm{PE}$ e em $6,9 \%$ do grupo GN. Contudo, a diferença entre os grupos não foi significativa. Por outro lado, a trombocitopenia não foi capaz de identificar a gravidade dos casos, pois nenhuma das grávidas incluídas apresentou contagem de plaquetas inferior a $100 \mathrm{~mL} / \mathrm{mm}^{3}$, limite considerado de gravidade. Apesar do número bem menor de mulheres envolvidas, nossos resultados são semelhantes aos encontrados por Matthews et al. ${ }^{7}$, que demonstraram trombocitopenia em $6,7 \%$ das gestantes normais, e por Miller e Nolan ${ }^{14}$, que, em estudo caso/controle do uso antenatal de cocaína, encontraram prevalência de trombocitopenia de $6,9 \%$ nos controles $(11 / 160)$.

Com relação ao volume médio das plaquetas, sua variação foi de 8,0-12,7 fL com média de 10,3 $\pm 1,2 \mathrm{fL}$ nas mulheres com gestação de evolução normal ao passo que nas mulheres com préeclâmpsia variou de 8,4 a 14,0 fL, com média de $11,0 \pm 1,2 \mathrm{fL}$. A diferença encontrada foi estatisticamente significativa. Järemo et al. ${ }^{3}$ encontraram valores de MPV mais baixos $(9,8 \pm 0,7 \mathrm{fL}$ e $8,8 \pm 1,2 \mathrm{fL}$ nos grupos PE e gestantes normais, respectivamente). Järemo e Sandberg-Gertzen ${ }^{15}$, em estudo no qual investigaram a densidade plaquetária e o MPV em portadores de doenças inflamatórias do intestino, demonstraram diminuição do MPV associado com atividade da colite ulcerativa e da doença de Crohn. Os valores encontrados para o MPV foram de 6,7 fL e 7,7 fL para pacientes com doença ativa e inativa, respectivamente, e de 7,6 fL para indivíduos normais incluidos como controles.

Nascimento ${ }^{8}$ considerou volume plaquetário de referência para o MPV de 8,0 a 9,6 fL, e macrocitose plaquetária um valor de MPV >9,7 fL. Nossos achados revelaram médias mais elevadas, com relação ao valor pré-estabelecido para macrocitose por Nascimento, tanto no grupo con- 
trole como no grupo $\mathrm{PE}$, porém mais expressiva nesse último. Chamamos a atenção para o fato de os grupos não serem comparáveis. Os indivíduos do estudo de Nascimento ${ }^{8}$ são de diferentes faixas etárias, inclusive crianças, e não de grávidas que compuseram nossa amostra. Além do mais, o equipamento utilizado é de outro fabricante, com outras especificações. A citação foi feita devido à inexistência de valores de referência no laboratório do HUJM, sendo esta uma das razões para a necessidade de um grupo controle constituído por grávidas normais.

É compreensivel que, em condições adversas, as plaquetas se modifiquem, alterando a sua forma e distribuição antes que a redução do número seja evidenciada, devido à ocorrência da coagulopatia de consumo, na maioria das vezes silenciosa pelo processo microangiopático.

Verificou-se correlação negativa significativa da contagem das plaquetas com o MPV. Esta idéia ficou reforçada no presente estudo, pelo achado de correlações também significativas terem sido evidenciadas com os outros parâmetros plaquetários: PDW e P-LCR. A presença de maiores volumes plaquetários, associada a menor contagem das plaquetas $/ \mathrm{mm}^{3}$, foi fato descrito por Nascimento et al. ${ }^{11}$. Outros autores também demonstraram correlação negativa de expressão moderada de $-0,46^{16}$.

A importância clínica de volumes plaquetários maiores tem sido relacionada com a presença de aumento da agregação plaquetária ${ }^{3,8,11}$. Outros comentam que a atividade das plaquetas traduzida pela agregação plaquetária não apresenta quadro característico durante a gravide ${ }^{16}$.

A densidade plaquetária está associada com reatividade plaquetária, e plaquetas de alta densidade são maiores e metabolicamente mais ativas que as de baixa densidade. Polanowska-Grabowska et al. ${ }^{17}$ demonstraram que o MPV diferiu de acordo com a subpopulação de plaquetas de baixa, intermediária e alta densidade, com valores de 6,0, 7,4 e 8,2 fL, respectivamente. Há escassez na literatura sobre alguns dados detectados, no presente estudo, sobre as fortes correlações positivas encontradas entre os demais parâmetros plaquetários entre si. Estas correlações se mostraram significativas entre o MPV e o PDW, entre MPV e P-LCR e também entre PDW e P-LCR.

Outros estudos sobre ativação plaquetária demonstraram que esta se encontrava mais aumentada nas portadoras de PE comparadas com grávidas normais, precedendo a sindrome clínica pelo mínimo de seis semanas ${ }^{18-20}$. Takeuchi et al. ${ }^{21}$ demonstraram maior agregação plaquetária nas mulheres com pré-eclâmpsia comparadas às grávidas normais, utilizando a adenosina como agen- te agregante em estudo in vitro. Segundo Dussé et al. ${ }^{22}$, é possível avaliar a ativação plaquetária pela determinação plasmática de marcadores especificos como a beta-tromboglobulina e o fator 4 plaquetário. Castro et al. ${ }^{23}$ concluíram que a falta de padronização de técnicas em estudos realizados tanto in vivo como in vitro da agregação plaquetária, associada à diversidade de agentes agregantes, têm contribuído para a heterogeneidade dos resultados encontrados. Ressalte-se ainda o fato de grávidas normais poderem apresentar trombocitopenia sem que repercussões clinicas sejam evidenciadas ${ }^{6,7}$.

Os resultados encontrados neste estudo de correlação negativa entre MPV e contagem das plaquetas foram demonstrados por outros autores $^{3,11,24}$. Contudo, estes autores não relataram a existência de semelhante correlação com os outros parâmetros do plaquetograma. No presente estudo demonstrou-se que semelhante correlação existe entre a contagem das plaquetas e os outros parâmetros plaquetários (PDW e P-LCR). Talvez, este achado possa ser explicado pelas significativas correlações demonstradas entre os demais parâmetros plaquetários entre si, detectando correlações positivas fortes entre MPV e PDW, entre MPV e P-LCR e entre PDW e P-LCR.

Conclui-se que não houve diferença entre os valores médios da contagem de plaquetas nas portadoras de $\mathrm{PE}$ comparadas às gestantes normais, ao passo que todos os demais parâmetros plaquetários foram significativamente mais elevados nas portadoras de $\mathrm{PE}$. As alterações aqui demonstradas na pré-eclâmpsia são importantes. Assim, a busca de marcador hematológico precoce na avaliação da pré-eclâmpsia é desejável. A aplicabilidade clínica desses novos parâmetros merece novos estudos.

\section{ABSTRACT}

Objective: to evaluate platelet parameters in normal and pre-eclamptic pregnant women.

Methods: a controlled cross-sectional study was carried out. The medical records of the women who had delivered in the Hospital Universitário Júlio Müller-Cuiabá/MT, from January 1, 2001 to July 31, 2002, were reviewed. The pregnant women were pre-selected based on their platelet parameters analyses performed after the 28th week of gestation. Two groups of study were analyzed: PE group (36 pre-eclamptic women) and NP group (58 normal pregnant women). The platelet parameters analyzed by the automated method were: platelet counts, mean platelet volume (MPV), platelet distribution width (PDW) and platelet - large cells ratio (PLCR). For statistical analysis the Student $t$-test and the chi- 
square test were used to compare the groups, and to evaluate the degree of dependence among the variables, the coefficient of determination was used $\left(\mathrm{r}^{2}\right)$. For all these tests, the significance level considered was $\mathrm{p} £ 0.05$.

Results: the platelet counts did not show difference between the two groups. However, all other platelet indices (MPV, $P D W$ and $P-L C R)$ were significantly higher in the PE group. The severity of the disease was documented in $91.7 \%$ of the pre-eclamptic women, despite the fact that none of the patients included had shown thrombocytopenia as a criterion of severity. Negative correlation was detected between the platelet counts and the other platelet parameters analyzed and there was positive correlation between MPV and PDW, $M P V$ and $P-L C R$, and PDW and P-LCR. Positive correlation was also observed between MPV, PDW and P-LCR, and the maximum systolic and diastolic blood pressures.

Conclusions: pre-eclampsia was associated with these platelet parameters, suggesting alterations of the platelet functions. The clinical applicability of these parameters, as early markers of severity of pre-eclampsia, needs more studies.

KEY WORDS: Pregnancy-induced hypertension. Preeclampsia. Platelet counts. Mean platelet volume. Thrombocytopenia.

\section{Referências}

1. Redman CW, Bonnar J, Beilin L. Early platelet consumption in pre-eclampsia. Br Med J 1978; 1:467-9.

2. Redman CW. Platelets and the beginnings of preeclampsia. N Engl J Med 1990; 323:478-80.

3. Järemo P, Lindahl TL, Lennmarken C, Forsgren H. The use of platelet density and volume measurements to estimate the severity of preeclampsia. Eur J Clin Invest 2000; 30:1113-8.

4. Bain BJ. Contagem de plaquetas. Valores de referência In: Bain BJ, editor. Células Sangüíneas: um guia prático. $2^{\mathrm{a}}$ ed. Porto Alegre: Artes Médicas; 1997. p. 31-51.

5. Mckenzie SB. Textbook of hematology. $2^{\text {nd }}$ ed. Philadelphia: Williams \& Wilkins; 1996. Automation in hematology. p. 635-57.

6. Failace R. Hemograma: manual de interpretação. $3^{\text {a }}$ ed. Porto Alegre: Artes Médicas; 1995. Plaquetograma. p. 173-8.

7. Matthews JH, Benjamin S, Gill DS, Smith NA. Pregnancy-associated thrombocytopenia: definition, incidence and natural history. Acta Haematol 1990; 84:24-9.

8. Nascimento MLP. Variações do volume plaquetário médio e faixa etária. NewsLab 2002; 10:127-42.

9. Järemo P. Computerised method for recording platelet density distribuition. Eur J Haematol 1995; 54:304-9.
10.Nascimento MLP, Silva LL, Fraga NTL, et al. Utilização do plaquetograma. Laes Haes 1998; 19:166-70.

11.Nascimento MLP, Silva LL, Fraga NTL. Volume plaquetário e suas relações com plaquetas, volume eritrocitário e hemácias. NewsLab 1999; 7:104-21.

12. Nascimento MLP. Contador hematológico penta 120 retic abx: histograma plaquetário e sua relação com os volumes eritrocitários e plaquetários. Laes Haes 2000; 22:180-94.

13. Ministério da Saúde. Gestação de alto risco. $3^{a}$ ed. Brasília; 2000. Sindromes hipertensivas da gravidez. p. 21-31.

14. Miller JM Jr, Nolan TE. Case-control study of antenatal cocaine and platelet levels. Am J Obstet Gynecol 2001; 84:434-7.

15.Järemo P, Sandberg-Gertzen H. Platelet density and size in inflammatory bowel disease. Thromb Haemost 1996; 75:560-1.

16.Rowland BL, Vermillion ST, Roudebush WE. Elevated circulating concentrations of platelet activating factor in preeclampsia. Am J Obstet Gynecol 2000; 183:930-2.

17.Polanowska-Grabowska R, Raha SR, Gear AR. Adhesion efficiency, platelet density and size. Br J Haematol 1992; 82:715-20.

18.Janes SL, Kyle PM, Redman C, Goodall AH. Flow cytometric detection of activated platelets in pregnant women prior to the development of preeclampsia. Thromb Haemost 1995; 74:1059-63.

19.Konijnenberg A, Stokkers EW, Joris AM, et al. Extensive platelet activation in preeclampsia compared with normal pregnancy: enhanced expression of cell adhesion molecules. Am J Obstet Gynecol 1997; 176:461-9.

20.Yoneyama Y, Suzuki S, Sawa R, Yoneyama K, Power GG, Araki T. Increased plasma adenosine concentrations and the severity of preeclampsia. Obstet Gynecol 2002; 100:1266-70.

21.Takeuchi T, Yoneyama Y, Suzuki S, Sawa R, Otsubo $\mathrm{Y}$, Araki T. Regulation of platelet aggregation in vitro by plasma adenosine in preeclampsia. Gynecol Obstet Invest 2001; 51:36-9.

22.Dussé LMS, Vieira LM, Carvalho MG. Revisão sobre alterações hemostáticas na doença hipertensiva específica da gravidez (DHEG). J Bras Patol Med Lab 2001; 37:267-72.

23. Castro RMF, Costa FS, Navarro PAS, Matthes ACS, Cunha SP. O sistema de coagulação na gravidez normal. Femina 2000; 28:29-32.

24.Agustino AM, Pérez PM, Rojas PG, Navarro JJF. Recuento da plaquetas y volumen plaquetario medio en una población sana. Rev Diego Biol 2002; 51:42-53.

Recebido em: 8/2/2004 Aceito com modificações em: 29/3/2004 